\title{
AVALIAÇÃO SILVICULTURAL DE ESPÉCIES FLORESTAIS NO ESTADO DE RORAIMA
}

\author{
Silvicultural Evaluation of forest species in the Roraima state
}

\author{
Marcelo Francia Arco-Verde" \\ Dalton Roberto Schwengber**
}

\section{Resumo}

O aumento na demanda por informações precisas e atualizadas de espécies florestais aptas para o plantio no Estado de Roraima, aliado à obrigatoriedade da reposição florestal por parte das indústrias madeireiras, levou à execução do presente estudo. O objetivo do trabalho foi selecionar espécies florestais de rápido crescimento para o Estado de Roraima. As espécies florestais foram escolhidas de acordo com a taxa de crescimento, boa adaptabilidade para crescimento a céu aberto; tecnologia disponível para produção de mudas e plantio; e demanda para produção de madeira para laminação, serraria, óleos essenciais, celulose e papel e fins energéticos. De acordo com as observações dos dados de crescimento em altura total, DAP e volume, podese citar o Paricá, Tachi-branco, Pará-pará e Morototó como as espécies nativas com potencial para programas silviculturais intensivos nas regiões de floresta estacional e úmida no estado de Roraima. Das espécies introduzidas, os clones 1270, 1232 e 321 de Eucalyptus urograndis apresentam os melhores resultados.

Palavras-chave: Plantios florestais, Silvicultura, Crescimento, Amazônia.

\section{Abstract}

The increasing claim for forestry information to choose species for plantation in the State of Roraima and the obligation of reforestation determined the necessity of this work. The central aim of this paper was to select rapid growth forest species to plant in Roraima. Forest species were chosen according to their growth rate, good adaptability to growth under light conditions, available technology to produce seedlings, wood demand for plywood, furniture, paper and fuelwood. Paricá, Tachi-branco, Pará-pará e Morototó are the native species with high possibility to be used in reforestation and research programs in humid region in Roraima State. Eucalyptus urograndis clones 1270, 1232 and 321 showed the best results.

Keywords: Forest stands; Silviculture, Growth rate, Amazon.

* Eng. Florestal, pesquisador da Embrapa Roraima. End: Caixa Postal 133, CEP 69301-970. Boa Vista-RR. Tel./fax (95) 626-7125, E-mail: arcoverd@cpafrr.embrapa.br

"* Eng. Agrônomo, pesquisador da Embrapa Roraima, E-mail: dalton@cpafrr.embrapa.br 


\section{Introdução}

O aumento na demanda de informações precisas e atualizadas de espécies florestais aptas para o plantio no Estado de Roraima, aliado à obrigatoriedade da reposição florestal por parte das indústrias madeireiras, levou à execução do presente estudo. O objetivo do trabalho foi cadastrar informações do desempenho de plantios florestais experimentais, assim como selecionar espécies florestais de rápido crescimento para as regiões de floresta estacional e úmida do Estado de Roraima.

\section{Material e métodos}

Os estudos foram realizados no campo experimental Confiança, localizado a $90 \mathrm{~km}$ de Boa Vista. As características edafoclimáticas foram as seguintes: vegetação de floresta, com clima ami (Koeppen), com precipitação anual média de 1900 $\mathrm{mm}$, onde a época chuvosa está definida no período de abril a setembro, sendo o mês de junho o mais chuvoso do ano, representando cerca de 19 $\%$ do total anual. As espécies estudadas foram: 1) 3 procedências de paricá (Schizolobium amazonicum), 2) mulateiro (Callycophyllum spruceanum), plantadas em julho de 1997; 3) sumaúma (Ceiba pentandra), 4) pinus (Pinus caribaea var. hondurensis), 5) freijó-cinza (Cordia goeldiana), 6) angelim-ferro (Dinizia excelsa), 7) 5 clones de eucaliptos (Eucalyptus urograndis), 8) pau-rainha (Centrolobium paraensis), 9) tachi-branco (Sclerolobium paniculatum), plantadas em maio de 1998; 10) 2 procedências de pará-pará (Jacaranda copaia), 11) teca (Tectona grandis), 12) cupiúba (Goupia glabra), 13) morototó (Scheffera morototoni), 14) cerejeira (Torresia acreana), 15) visgueiro (Parkia multijuga), 16) cedrorana (Cedrelinga cataeniformis), 17) castanha-do-Brasil (Bertholletia excelsa), plantadas em maio de 1999; e 18) virola (Virola surinamensis) e 19) cardeiro (Scleronema micranthum), plantadas em maio de 2000.

As espécies florestais foram escolhidas de acordo com os seguintes critérios: a) taxa de crescimento de rápido a médio, com pelo menos 0,60 $\mathrm{m}$ /ano de incremento em altura em plantios experimentais/comerciais e em regeneração natural; b) boa adaptabilidade para crescimento a céu aberto; c) tecnologia disponível para produção de mudas e plantio; e d) demanda para produção de madeira para laminação, serraria, óleos essenciais, celu- lose e papel e fins energéticos.

A coleta das sementes de cada espécie foi realizada buscando uma boa qualidade genética, sendo selecionadas, quando possível, pelo menos 20 árvores matrizes para cada espécie, representando, assim, uma procedência/população. Em alguns casos o número de árvores onde foram coletadas as sementes foi inferior a 20. Isto ocorreu devido à sazonalidade da fase de produção de sementes de algumas espécies, dificuldade em alcançar a distância mínima entre as matrizes, acesso e elevado custo para buscar novas áreas de coleta. As árvores matrizes foram amostradas ao acaso na população, tomando-se o cuidado de manter uma distância mínima de pelo menos duas vezes a altura da árvore, evitando-se coletar sementes de indivíduos aparentados e garantindo maior variabilidade genética nas futuras gerações.

O preparo do solo consistiu numa roçada seguida de queimada da capoeira de aproximadamente 5 anos de idade. A adubação foi de 60 $\mathrm{g}$ de superfosfato triplo/cova. O plantio das espécies destinadas à produção de madeira para a serraria foram plantadas num espaçamento de $3 \times 4$ $\mathrm{m}\left(12 \mathrm{~m}^{2}\right)$; e as espécies para energia e celulose foram plantadas no espaçamento de $3 \times 2 \mathrm{~m}$ (6 $\mathrm{m}^{2}$ ). Cada parcela consistiu de um total de 81 plantas $(9 \times 9)$, com 49 plantas úteis $(7 \times 7)$.

Foram analisados anualmente dados de sobrevivência, crescimento em diâmetro (DAP), altura total, forma do fuste, danos causados por pragas e/ou doenças, propriedades químicas e físicas do solo.

Os estudos iniciaram em 1996, com os primeiros plantios sendo realizados em junho de 1997. A época chuvosa está definida no período de abril a setembro, sendo o mês de junho o mais chuvoso do ano, representando aproximadamente $19 \%$ da precipitação total anual.

\section{Resultados}

Das análises químicas do solo, pode-se observar baixos níveis de fertilidade. $\mathrm{O} \mathrm{pH}$ foi de 4,5 com valores de alumínio de $0,82 \mathrm{cmol}_{\mathrm{c}} \cdot \mathrm{dm}^{-3}$. Os níveis de matéria orgânica e macroelementos foram os seguintes: matéria orgânica $=23,2 \mathrm{~g} \cdot \mathrm{dm}^{-3}$; fósforo = 1,8 mg.dm $\mathrm{dm}^{-3}$; potássio $=28,6 \mathrm{mg} \cdot \mathrm{dm}^{-3}$; cálcio $=0,49 \mathrm{cmol}_{\mathrm{c}} \cdot \mathrm{dm}^{-3} ;$ e magnésio $=0,26$ $\mathrm{cmol}_{\mathrm{c}} \cdot \mathrm{dm}^{-3}$. Tais resultados já eram esperados, uma 
vez que estes solos representam as características edáficas de baixa fertilidade da região.

Da análise física do solo, observaram-se teores de argila, silte e areia de 22,5\%, 20,7\% e 56,6\%, respectivamente, na profundidade de $0-20 \mathrm{~cm}$. De forma geral, houve maior concentração de argila nas camadas mais profundas e de areia nas camadas superiores do solo. Quanto ao silte, este se manteve praticamente constante nas diferentes profundidades. Na profundidade de $20-40 \mathrm{~cm}$ observaram-se os teores de argila, silte e areia de $29,8 \%, 18,5 \%$ e $51,5 \%$, respectivamente.

Durante a avaliação de pragas, ocorreu a presença de Costalimaita sp., no início do período chuvoso, e formigas cortadeiras (Atta sp.) nas áreas de Eucalyptus urograndis. O ataque de Costalimaita sp. pode causar total desfolhação das árvo- res, prejudicando seriamente o crescimento do plantio.

A tabela 1 apresenta os resultados da altura, DAP e volume das espécies florestais em estudo, identificando os clones, procedências, função de cada espécie e a época de plantio. O melhor crescimento pode ser observado nos dados de Eucalyptus urograndis, clone 1270, como espécie introduzida, alcançando 292,77 $\mathrm{m}^{3}$ aos 2,5 anos de idade; e nos resultados de Paricá, procedência de Rondônia, como espécie nativa, atingindo $275,62 \mathrm{~m}^{3}$ aos 3,5 anos de idade. Outro bom resultado foi observado no crescimento em altura, dap e volume de Tachi-branco, com valores de $7,98 \mathrm{~m}, 9,65 \mathrm{~cm}$ e $73,29 \mathrm{~m}^{3}$ respectivamente aos 2,5 anos de idade.

TABELA 1 - Características das espécies avaliadas em área de floresta do estado de Roraima (medições de 2001) Table 1 - Characteristics of tree species from a forest area in the state of Roraima (measurements of 2001)

\begin{tabular}{|c|c|c|c|c|c|c|c|c|c|}
\hline \multirow{3}{*}{ Espécies } & \multirow[b]{2}{*}{ Clones/ } & \multirow{2}{*}{\multicolumn{2}{|c|}{ Função }} & \multirow{2}{*}{\multicolumn{3}{|c|}{$\begin{array}{l}\text { Ano de plantio } \\
\text { (No. de plantas) }\end{array}$}} & \multicolumn{3}{|c|}{ Média } \\
\hline & & & & & & & Altura & DAP & Volume \\
\hline & Proc. & Madeira & Energia & 97 & 98 & 99 & $(\mathrm{~m})$ & $(\mathrm{cm})$ & $\left(m^{3} \cdot h a^{-1}\right)$ \\
\hline Andiroba & & + & & 29 & & & 3,80 & 3,95 & 4,41 \\
\hline Angelim & & + & & & 64 & & 3,20 & 4,36 & 4,35 \\
\hline Castanheira & & + & & & & 46 & 1,85 & 3,20 & 0,92 \\
\hline Cerejeira & & + & & & & 44 & 2,35 & 4,70 & 3,00 \\
\hline \multirow[t]{5}{*}{ Eucaliptos } & 103 & & + & & 49 & & 12,23 & 9,48 & 96,30 \\
\hline & 1232 & & + & & 43 & & 17,92 & 10,87 & 183,50 \\
\hline & 1270 & & + & & 42 & & 20,70 & 12,54 & 292,27 \\
\hline & 1341 & & + & & 46 & & 10,88 & 7,58 & 55,03 \\
\hline & 321 & & + & & 39 & & 14,76 & 9,36 & 118,21 \\
\hline Morototó & & + & & & & 47 & 4,07 & 8,48 & 13,68 \\
\hline Mulateiro & & + & & 22 & & & 6,03 & 4,18 & 6,49 \\
\hline \multirow[t]{2}{*}{ Pará-pará } & PA & + & & & & 31 & 3,87 & 5,56 & 6,31 \\
\hline & RR & + & & & & 49 & 5,91 & 8,16 & 22,88 \\
\hline \multirow[t]{3}{*}{ Paricá } & $A C$ & + & & 40 & & & 20,70 & 14,94 & 202,42 \\
\hline & PA & + & & 43 & & & 20,88 & 15,95 & 232,52 \\
\hline & RO & + & & 46 & & & 22,25 & 16,87 & 275,62 \\
\hline Pau rainha & & + & & & 45 & & 4,17 & 4,29 & 4,19 \\
\hline Samaúma & & + & & & 48 & & 4,18 & 8,67 & 14,85 \\
\hline Tachi branco & & & + & & 26 & & 7,98 & 9,65 & 73,29 \\
\hline Teca & & + & & & & 48 & 6,71 & 5,97 & 11,68 \\
\hline Visgueiro & & + & & & & 44 & 3,03 & 6,58 & 6,24 \\
\hline
\end{tabular}

Os valores referentes ao incremento médio anual em volume (IMA) das espécies florestais avaliadas são apresentados na Tabela 2 e Figura 1. Das espécies plantadas em 1997, avaliadas com 3,5 anos de idade, o Paricá procedente de Rondô- nia obteve IMA de $68,91 \mathrm{~m}^{3}$, com médias em seus extremos inferior e superior de $63,18 \mathrm{~m}^{3}$ e 74,64 $\mathrm{m}^{3}$, respectivamente. Andiroba e Mulateiro apresentaram um IMA até 50 vezes menor que o Paricá. Das espécies plantadas em 98, com 2,5 anos 
de idade, os de Eucalyptus urograndis 1270, 1232 obtiveram os melhores resultados $\left(97,42 \mathrm{~m}^{3}\right.$ e 61,17 $\mathrm{m}^{3}$, respectivamente). Entretanto, deve-se também destacar o clone 103, com 32,10 $\mathrm{m}^{3}$, e Tachi-branco, com IMA de $24,43 \mathrm{~m}^{3}$. Do plantio realizado em 1999, com idade de 1,5 ano, o melhor IMA foi observado nas árvores de Pará-pará, procedência Roraima, com 11,44 $\mathrm{m}^{3}$ seguido de Morototó (6,84 $\left.\mathrm{m}^{3}\right)$ e Teca $\left(5,84 \mathrm{~m}^{3}\right)$. Os valores mais baixos foram medidos nos indivíduos de Castanha-do-Brasil $\left(0,46 \mathrm{~m}^{3}\right)$.

TABELA 2 - Incremento médio anual (IMA) em volume.ha-1 das espécies. O valor de $25 \mathrm{~m}^{3} \cdot \mathrm{ha}^{-1}$ ano $^{-1}$ corresponde ao parâmetro de comparação entre as espécies

Table 2 - Annual mean increment (AMI) in volume.ha of the species. The value of $25 \mathrm{~m}^{3} . \mathrm{ha}^{-1} \mathrm{year}^{1}$ corresponds to the parameter for comparison between the species

\begin{tabular}{|c|c|c|c|c|c|c|}
\hline Ano & Espécies & Clo/Pro & IMA & Média-1,96*EP & Média+1,96*EP & $25 \mathrm{~m}^{3}$. ano ${ }^{-1}$ \\
\hline \multirow{5}{*}{97} & Andiroba & & 1,10 & 0,44 & 1,76 & [o] \\
\hline & Mulateiro & & 1,62 & 0,83 & 2,42 & [0] \\
\hline & Paricá & $A C$ & 50,61 & 45,33 & 55,88 & {$[+]$} \\
\hline & & PA & 58,13 & 51,83 & 64,43 & {$[+]$} \\
\hline & & RO & 68,91 & 63,18 & 74,64 & {$[+]$} \\
\hline \multirow{9}{*}{98} & Angelim & & 1,47 & 1,01 & 1,94 & [0] \\
\hline & Eucaliptus & 103 & 32,10 & 29,17 & 35,03 & {$[+]$} \\
\hline & & 1232 & 61,17 & 56,10 & 66,23 & {$[+]$} \\
\hline & & 1270 & 97,42 & 85,57 & 109,27 & {$[+]$} \\
\hline & & 1341 & 18,34 & 16,60 & 20,09 & [0] \\
\hline & & 321 & 39,40 & 33,63 & 45,18 & {$[+]$} \\
\hline & Pau rainha & & 1,40 & 1,03 & 1,76 & [0] \\
\hline & Samaúma & & 4,95 & 4,13 & 5,77 & [0] \\
\hline & Tachi branco & & 24,43 & 18,03 & 30,83 & [0] \\
\hline \multirow{7}{*}{99} & Castanheira & & 0,46 & 0,37 & 0,56 & [0] \\
\hline & Cerejeira & & 1,50 & 1,02 & 1,98 & [0] \\
\hline & Morototó & & 6,84 & 5,72 & 7,96 & [0] \\
\hline & Pará-pará & PA & 3,15 & 2,17 & 4,14 & [0] \\
\hline & & $\mathrm{RR}$ & 11,44 & 8,53 & 14,35 & [0] \\
\hline & Teca & & 5,84 & 4,76 & 6,92 & [0] \\
\hline & Visgueiro & & 3,12 & 2,55 & 3,69 & [0] \\
\hline
\end{tabular}

FIGURA 01 - Comparação das espécies florestais com crescimento acima e abaixo do incremento médio anual (IMA) de $25 \mathrm{~m}^{3}$

Figure 01 - Comparison of the forest species with growth above and below to the annual mean increment (AMI) of $25 \mathrm{~m}^{3}$

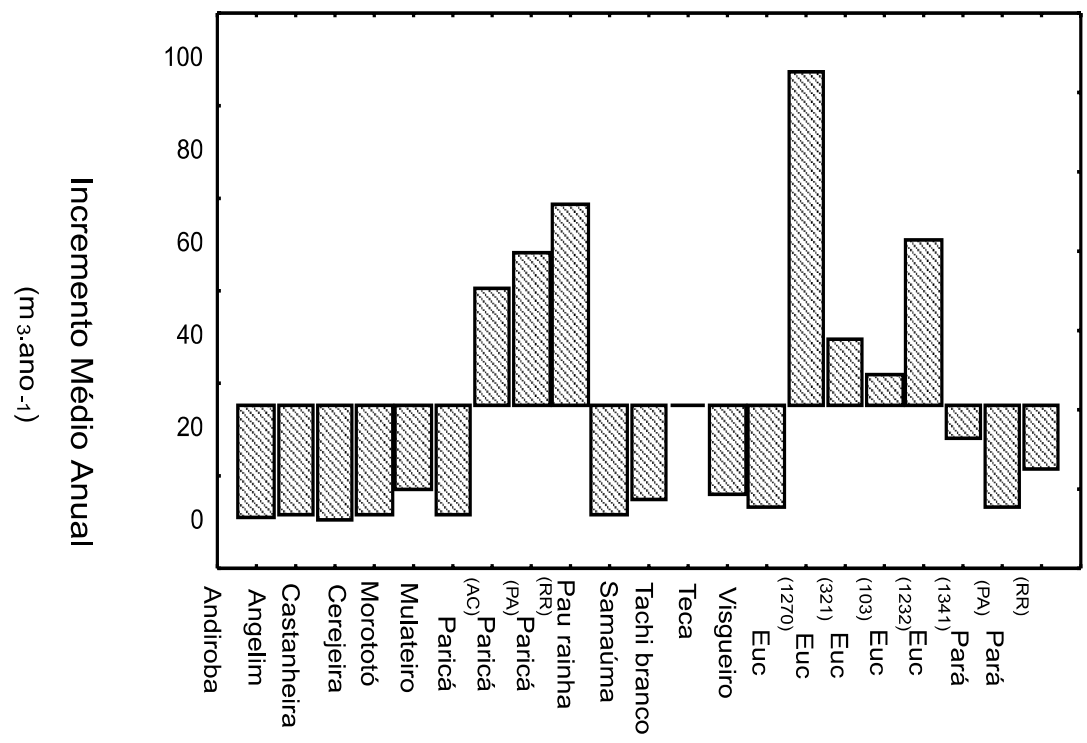


A fim de simular a dispersão dos dados de crescimento em indivíduos de uma mesma população, realizou-se a simulação do volume dos indivíduos de cada clone de Eucalyptus urograndis (Figura 2).

FIGURA 2 - Simulação da dispersão em volume $\left(\mathrm{m}^{3} \cdot \mathrm{ha}^{-1}\right)$ com dados de clones de Eucalyptus urograndis Figure 2 -Simulation of the dispersion in volume $\left(\mathrm{m}^{3} \cdot \mathrm{ha^{1 }}\right)$ using data from clones of the Eucalyptus urograndis

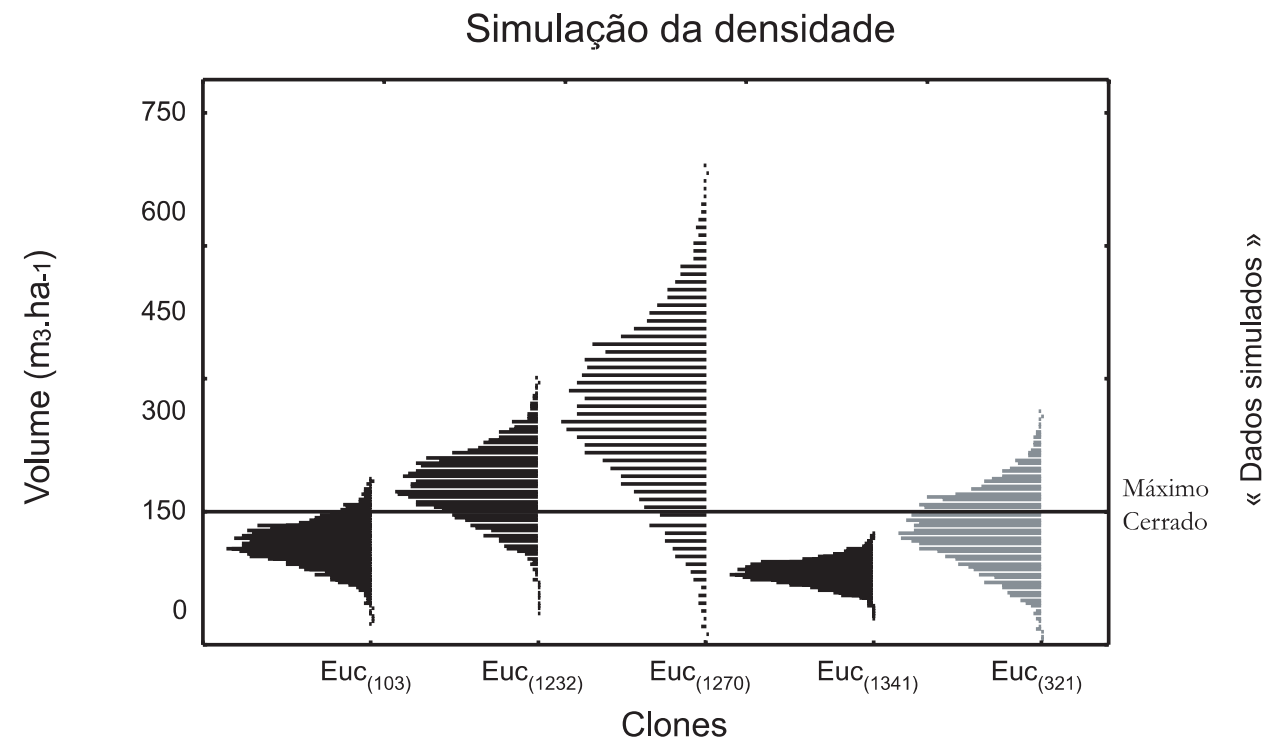

A maior variação existente nas populações ocorreu no clone mais produtivo, o clone 1270, enquanto o clone 1341, o de menor produtividade, apresentou a menor dispersão.

\section{Discussão e considerações finais}

De acordo com as observações dos dados de crescimento em altura total, DAP e volume, pode-se citar o Paricá, Tachi-branco, Pará-pará e Morototó como as espécies nativas com potencial para estarem presentes em programas silviculturais intensivos nas regiões de floresta estacional e úmida no estado de Roraima. Das espécies introduzidas, os clones 1270, 1232 e 321 de Eucalyptus urograndis apresentam os melhores resultados de crescimento. É importante lembrar que resultados tão satisfatórios foram obtidos somente com a aplicação de $60 \mathrm{~g}$ de superfosfato triplo em cada cova da muda plantada.

Há a necessidade de realizar novos estudos com diferentes preparos de área e níveis de fertilização para as espécies que apresentaram rá- pido crescimento e, principalmente para as espécies de alto valor comercial que não demonstraram bons resultados de crescimento, tais como Castanha-do-Brasil, Andiroba, Mulateiro, Pau-rainha e Sumaúma.

\section{Referências}

Convênio SUDAM/OEA/PROVAM. Atualização dos estudos básicos do Vale do Rio Branco (Estado de Roraima): Clima. Belém: SUDAM: OEA: PROVAN, 1993. v.1.

LIMA, R.M.B. et al. Zoneamento edafo-climático para plantios de espécies florestais de rápido crescimento na Amazônia. In: RESULTADOS (I fase emergencial e fase): Programa piloto para a proteção das florestas tropicais do Brasil. Manaus, AM.: [s. n.], 1999. p.309 - 331. 